\title{
Measurements of Intrabeam Scattering Rates below Transition in the Fermilab Antiproton Accumulator*
}

\author{
C. Bhat, L. K. Spentzouris and P. L. Colestock \\ Fermi National Accelerator Laboratory \\ P. O. Box 500, Batavia, IL 60510, USA
}

\begin{abstract}
An important factor in the successful operation of the Antiproton Recycler Project at Fermilab is the rate at which intrabeam scattering heats the stored beam in each plane. This machine will operate below transition energy. An application of classical intrabeam scattering theory for such a machine indicates that transverse or longitudinal cooling might occur, rather than heating, under certain conditions. However, this effect has not been studied experimentally. Hence, we have undertaken a study of the heating/cooling rates below transition in a coasting antiproton beam in the Fermilab Accumulator Ring, in an attempt to verify the theoretical models in this regime. This storage ring employs a variety of stochastic cooling systems which can be used to alter the ratio of emittances in each plane as the initial conditions for our experiment. We have used both a conventional free-expansion technique as well as a longitudinal-echo-decay-rates technique to determine the scattering rates. In this paper, we present a preliminary analysis of our data and its comparison with various intrabeam scattering models below transition.
\end{abstract}

\section{INTRODUCTION}

It is a well-known theoretical result that inter-particle collisions cause an exchange of energy from the directed motion of relativistic beam above transition into thermal energy in all three planes of motion. [1], [2] A body of experimental results [3] has confirmed the general character of this model. It is also expected that below the transition energy, the behavior of the beam particles approaches that of a normal gas. Namely each degree of freedom exchanges energy with another according to its relative temperature. In particular, it is possible to expect cooling of the horizontal plane below transition, as the longitudinal plane in typical situations is initially colder than either of the transverse planes. This behavior has not yet been observed in a high-energy storage ring.

In the Fermilab Recycler, a stored beam of antiprotons will exist below the transition energy of that device, and it is important to understand the effects that intrabeam scattering will have on emittance growth, as this fixes the requirement for cooling of the stored beam. To elucidate this issue, we have carried out a series of experiments in the Fermilab Antiproton Accumulator below transition, and com-

\footnotetext{
* Operated by the Universities Research Association,Inc, under contract with the U.S. Department of Energy
}

pared the results with the existing models. In this paper we discuss both the experimental results and the comparison with theory.

\section{EXPERIMENTAL RESULTS}

The approach taken in this work was to study the free expansion of the stored beam beginning from a variety of initial conditions, facilitated by the flexible stochastic cooling system on the Accumulator. In addition, longitudinal echoes [4] were used to provide a second measurement of the longitudinal diffusion. Both methods are expected to be valid over a range of scattering times, but the echo method is in principle valid to much longer decay times than where free expansion measurements can readily yield useful results. For these experiments, a suitable lattice for below-transition operation was prepared, along with procedures for decelerating the stored beam. The lattice used had a transition gamma of 5.42, and the beam was successfully decelerated to a gamma of 4.86. Beam losses during deceleration prevented operation at lower energies. While this energy is not sufficiently far below transition to clearly demonstrate transverse cooling, it extends into a new regime of the scattering models that has not received detailed study, and will help confirm the applicability of the scattering models below transition.

The measurement technique consisted of observing the free expansion of the beam with all cooling systems turned off, starting from a variety of initial conditions which spanned the achievable transverse and longitudinal emittance spaces. Emittances were monitored continuously by measuring the spectral width of the longitudinal Schottky noise or by a similar measurement of the transverse fluctuations. Since the translation of this data into emittance values requires detailed knowledge of the twiss parameters, some systematic error is expected to be introduced in this step, as these parameters were not directly measured in these experiments. Longitudinal echoes were also used as a second check of the longitudinal diffusion rates, especially because the accuracy of the echo measurement extended to the ultra-weak diffusion regime $\left(<0.1 \mathrm{hrs}^{-1}\right)$ where free expansion measurements are limited by measurement error. Echo measurements were carried out at the beginning and end of each free expansion measurement to aid in corroboration of the two methods. Typical expansion data is shown in Fig. 1. Typical echo decay data is shown in Fig. 2. 

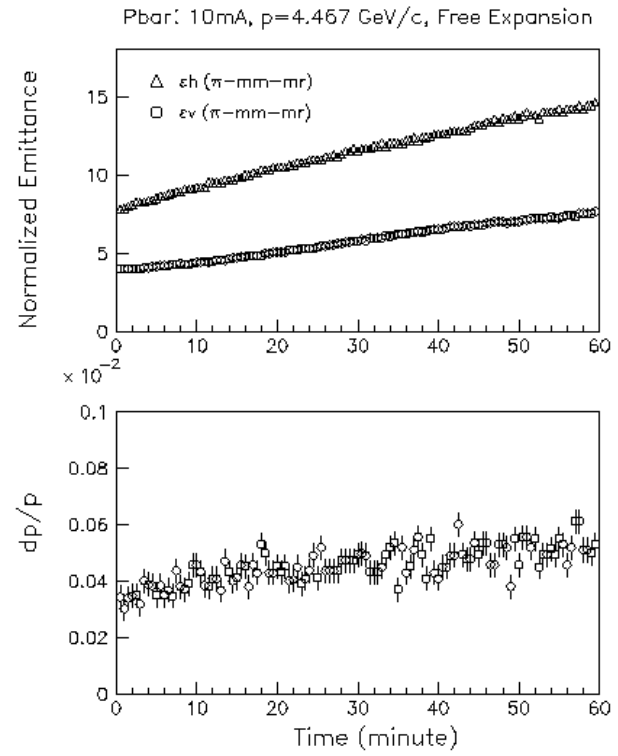

Figure 1: Free expansion measurements. All cooling systems are switched off for these measurements. The growth time is found by fitting a low-order polynomial to the emittance evolution with time.

\subsection{Comparison with Theory}

The theoretical predictions are based on the complete intrabeam scattering theory [2] as applied to the full lattice model of the Accumulator below transition. The calculation of the growth times depends on detailed knowledge of the twiss parameters of the machine, which were not directly measured for these experiments, but were calculated using the MAD code. The results of this calculation for these experiments are shown in Fig. 3, and are subject to an estimated $30 \%$ systematic error. This corresponds to a similar error in the calculated growth times. In addition, measurements of the beam emittances were subject to a systematic error estimated at $20 \%$. While this leaves considerable lattitude in comparing the theory with the experiment, the systematic character of the error does permit a comparison of the scaling of the scattering rates.

For the lattice shown in Fig. 3 , the growth rates evaluated locally for an uncoupled lattice are shown in Fig. 4. The comparison of the experimental data with the theory for growth in the longitudinal plane is shown in Fig. 5. We find an overall favorable agreement in the scaling over a factor of one hundred in the growth rate, although adjust-
Longitudinal Echo Decay

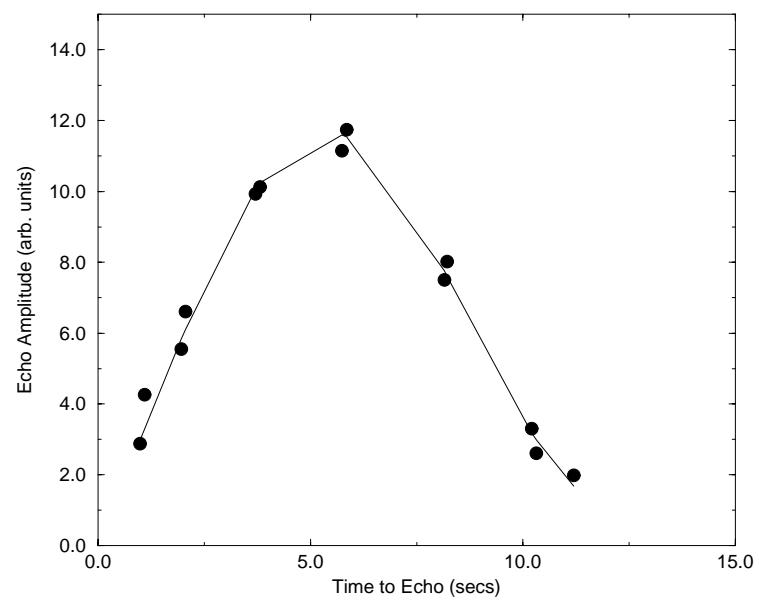

Figure 2: Longitudinal echo decay measurements. All cooling systems are switched off for these measurements. The growth time is found by fitting a two-parameter model (solid line) to the measured decay envelope (points) which yields both the amplitude and the inverse scattering time.

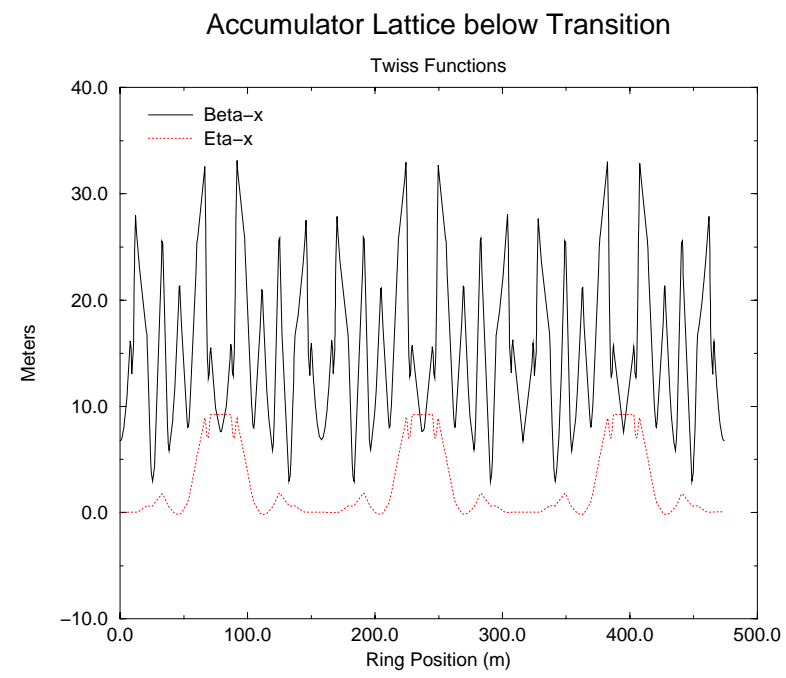

Figure 3: Below transition lattice as calculated by MAD. Similar functions are found for the vertical plane.

ments in both the transverse and longtiudinal emittances did have to be made to achieve this agreement, as noted. Since the vertical growth for an uncoupled machine is negligibly small, the observed equal growth rates in each plane suggest strong coupling. [5] While the source of coupling in the machine was not measured, and hence could not be calculated, the observed equal growth rates in the vertical and horizontal planes suggests $100 \%$ coupling existed. As such, a useful approach is then to average the emittances in the two planes for calculating the growth rates. Hence, the transverse emittances were assumed to be equal, and re- 
duced from the calculated values by $30 \%$. The longitudinal emattance was reduced by $25 \%$. Both of these assumptions are considered consistent with the expected accuracy of the calculated twiss functions. We note that there is excellent agreement between the free expansion data and the echoes where the data overlaps. Moreover, the echo data extends far into the weak diffusion regime $\left(<0.1 \mathrm{hrs}^{-1}\right)$.

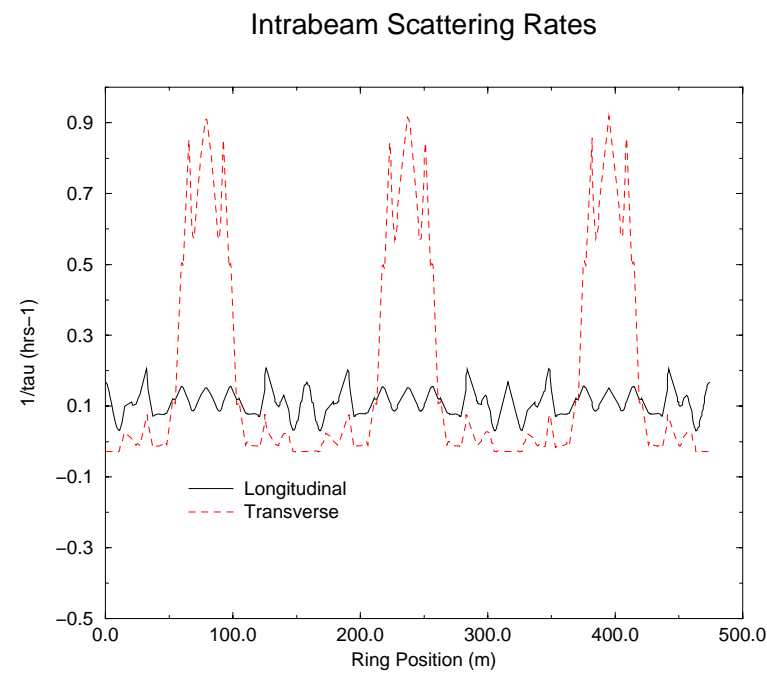

Figure 4: Intrabeam scattering rates evaluated locally around the ring for a specific set of measured emittances.

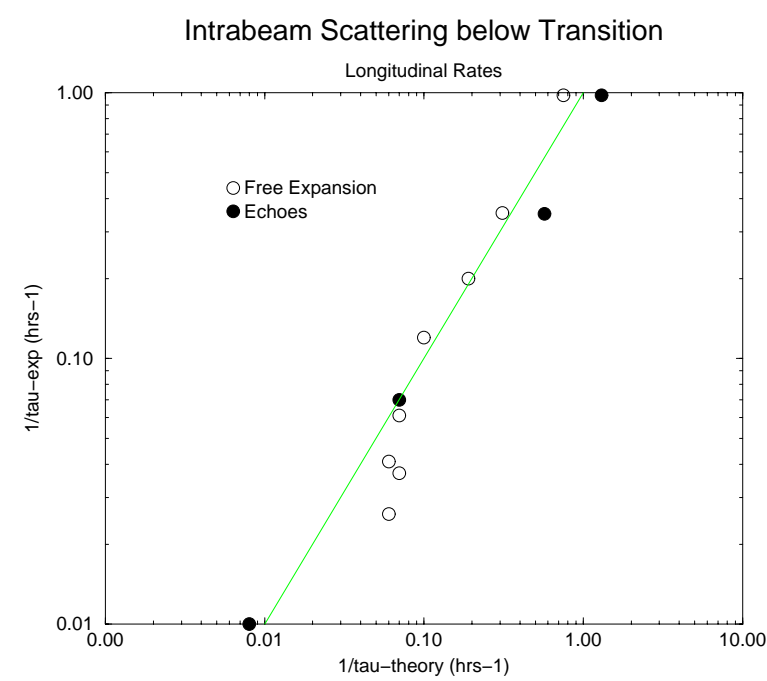

Figure 5: Comparison of the theory to experiment for longitudinal scattering rates. The dashed lines represents agreement.

The corresponding transverse data is shown in Fig. 6 as derived from free expansion data. Here the correspondence to the theory is less satisfactory, however there are expected uncertainties of the twiss functions and transverse emittances, in addition to measurement errors at long growth times. As such, the overall scaling can be viewed as roughly consistent with the theoretical model.

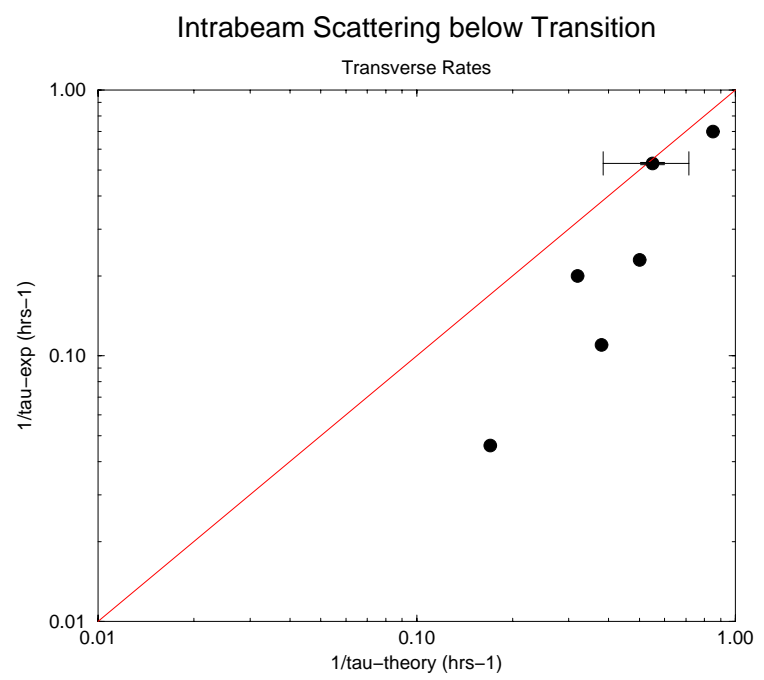

Figure 6: Comparison of the theory to experiment for transverse scattering rates.

\subsection{Discussion}

We find very good agreement between the experimental intrabeam scattering data and the models provided the following adjustments are made. First, the transverse emittances are reduced by $30 \%$ to accomodate a systematic uncertainty in the emittance measurements. Second, coupling was invoked to explain the observed heating of the vertical plane, as noted above. Finally, a 20\% reduction of the longitudinal emittance was assumed. Since, neither coupling nor emittance measurements were verified by an alternative method, we have no way of directly corroborating these assumptions. However, once correction factors are determined and applied to the data, the observed agreement of the scaling between theory and experiment suggests that the model is likely correct. We conclude that the existing scattering models are at least consistent with the experimental data in the regime around and below transition.

\section{REFERENCES}

[1] A. Piwinski, Proc. 9th Int. Conf. on High Energy Accelerators, Stanford, p. 406, (1974)

[2] J. D. Bjorken and S. K. Mtingwa, Particle Accelerators, Vol. 13, p. 115 (1983)

[3] M. Martini, CERN PS/84-9 (1984)

[4] L. K. Spentzouris, et al., Phys. Rev. Lett. 76, 4 (1996)

[5] A. Piwinski, DESY 90-113, Sept. (1990) 